\title{
ADICCIÓN A LA PORNOGRAFÍA: INTERFERENCIA ATENCIONAL Y GRAVEDAD DEL CONSUMO
}

\author{
Cervigón-Carrasco, $\mathrm{V}$ \\ Dpto. de Psicología Básica, Clínica y Psicobiología \\ Universitat Jaume I, Castellón \\ veronica.cervigon@uji.es \\ Castro-Calvo, J \\ Departamento de Personalidad, Evaluación y Tratamientos Psicológicos \\ Universitat de València, Spain \\ Gil-Juliá, B \\ Departamento de Personalidad, Evaluación y Tratamientos Psicológicos \\ Universitat de València, Spain \\ Giménez-García, C \\ Dpto. de Psicología Básica, Clínica y Psicobiología \\ Universitat Jaume I, Castellón \\ Ballester-Arnal, $\mathbf{R}$ \\ Dpto. de Psicología Básica, Clínica y Psicobiología \\ Universitat Jaume I, Castellón
}

Fecha de Recepción: 6 Febrero 2019

Fecha de Admisión: 30 Abril 2019

\section{RESUMEN}

Introducción: El aumento del uso de las Tecnologías de la Información y la Comunicación (TICS) e Internet en nuestra sociedad ha potenciado la aparición de nuevas formas de adicción. Una de las más relevantes por sus implicaciones sociosanitarias sería la adicción al cibersexo, y más en concreto, el visionado excesivo de pornografía online. Numerosos estudios apuntan que, en determinadas personas, el visionado de pornografía puede sobrevenir excesivo e incontrolado, generando graves problemas en las diferentes áreas de la vida. Por ello, es importante explorar aquellos factores que actúan como predisponentes o mantenedores de este problema. Se postula que uno de ellos podría ser la capacidad que tiene el material pornográfico para atraer y consumir recursos atencionales (cue reactivity), generando una interferencia en la atención a otros estímulos relevantes. Por tanto, el objetivo de este trabajo sería explorar la relación entre la interferencia atencional inducida por la exposición a contenidos pornográficos y la severidad de dicha conducta. Método: A fin de evaluar dichos sesgos atencionales, se desarrolló una tarea experimental que se aplicó a participantes con edades comprendidas entre los 18 y 35 años. La tarea experimental consistía en res- 
ponder a una tarea Stroop al mismo tiempo que los participantes eran expuestos a cuatro tipos de video (pornográfico, serie de televisión, videojuego y contenido poco interactivo -un vídeo de un hombre leyendo el periódico-). Los participantes recibían la premisa de ignorar los videos y centrarse en responder de la forma más rápida y certera a la tarea Stroop. Resultados: El nivel de interferencia atencional inducida por la pornografía en comparación a la inducida por los otros tipos de contenidos se evalúo a través del contraste de los tiempos de reacción medios y los aciertos y errores de cada ensayo Stroop. Dichos resultados, donde se aprecian importantes diferencias entre las distintas condiciones experimentales, se exponen de forma detallada durante la presentación del presente trabajo. Conclusiones: Este estudio confirma la capacidad de distintos contenidos multimedia (entre ellos, la pornografía) para atraer y consumir recursos atencionales, así como la relación entre la capacidad de interferencia atencional y su potencial adictivo. Así, resulta primordial plantear nuevas aproximaciones clínicas que den relevancia a este aspecto tanto a nivel de evaluación como a nivel de tratamiento e intervención directa.

Palabras clave: adicción a la pornografía; interferencia atencional; jóvenes

\section{ABSTRACT}

Pornography addiction: attention interference and severity of behavior. Introduction: The increased use of Information and Comunication Tecnologies (ICT) and internet in our society has boosted new forms of addiction. Because of its public health concerns, one of the most important is cybersex addiction, and in particular excessive and problematic pornography consumption. Large number of studies pointed out that in some people, pornography viewing can be excessive and uncontrolled, creating several problems in different areas of life. For this reason, it is important to explore the underlying factors and maintainers in this problem. It is postulated that one of these factors could be the pornographic content ability to attract and consume attention resources (cue reactivity). The purpose of this study is to explore the relation between the attention interference created by the pornography contents viewing and severity of this behaviour. Method: To assess this attentional bias, we developed and applied an experimental task to a group of participants whose age oscillates between 18 and 35 years old. The experimental task was based on a Stroop task paradigm: participants concurrently answer to this attentional task when they were exposed to four types of contents (pornographic video, sitcom, video game and a low interactive content -a man reading a newspaper-). Participants received the premise of ignoring videos and focusing on answer quickly and accurately the Stroop task. Results: Level of attention interference created by pornography compared to other contents was assessed through the comparison between average reaction times and right answer and mistakes of each trial. These results, where we showed significant differences between the experimental conditions, will be exposed in detail during the presentation of the study. Conclusions: This study supports, on the one hand, the big ability of the different multimedia contents (among them, pornography), to attract and consume attentional resources; on the other hand, it also highlights the strong relation with the ability of attention interference that have their addictive potential. Therefore, it is important to include new clinical approaches that address this aspect at different levels: prevention, assessment, and treatment.

Keywords: pornography addiction; attentional bias; young people

\section{INTRODUCCIÓN}

En los últimos años, las TICs (Tecnologías de la Información y Comunicación), y especialmente Internet, han adquirido un papel fundamental en la sociedad debido a su gran expansión y generalización, que además sigue en aumento (Griffiths, 2010; Cooper, McLoughlin \& Campbell, 2000). Dicho crecimiento del uso de Internet junto al hecho de que éste está provocando cambios en los 
patrones de comportamiento de sus usuarios han propiciado que la red y las múltiples actividades online derivadas se hayan erigido como objeto de interés en la literatura científica de los últimos años (Serrano \& Cuesta, 2018; Ballester, 2006)

Cuando se han analizado los patrones de uso de internet y su actividad, se ha encontrado que parte de las actividades más populares en internet están vinculadas con el sexo y constructos relacionados (McCullough, 2015). En esta línea, el sexo es la temática con la que se vinculan más búsquedas online diarias (Griffiths, 2010; Griffiths, 2000). Cooper, McLoughlin \& Campbell (2000) encuentran que hasta 9 millones de usuarios de internet usan internet diariamente con fines relacionados con el sexo y el campo de la sexualidad, actividades conocidas como OSAs (online sexual activities) (Cooper, Scherer, Boies \& Gordon, 1999; Griffiths, 2010). Existe gran variedad de OSAs, entre las que destacan: la búsqueda de materiales educativos y académicos, la compra-venta de bienes sexuales, la búsqueda de material para uso y disfrute (vinculado a la masturbación y pornografía) 0 la búsqueda de parejas sexuales entre otras (Griffiths, 2010; Ballester, 2006; Griffiths, 2000). Sin embargo, la investigación actual se centra especialmente en el subtipo de OSA que se conoce como cibersexo, cuya finalidad principal característica es que su objetivo es obtener satisfacción y gratificación sexuales (Dorton \& Gast, 2007), y que implica mayoritariamente actividades como la búsqueda de parejas sexuales, la participación chats-rooms sexuales y la visualización de pornografía online (Wéry \& Billieux, 2017). Precisamente esta última, se considera la actividad sexual online más popular (Wéry \& Billieux, 2017;Laier, Pawlikowski, Pekal, Schulte \& Brand, 2013).

A pesar de los múltiples beneficios para el desarrollo y el crecimiento sexual vinculados a esta actividad sexual online y que se describen en múltiples estudios (Serrano \& Cuesta, 2018; Laier, Pawlikowski, Pekal, Schulte \& Brand, 2013; Grov, Bamonte, fuentes, Parsons, Bimbi \& Morgenstern, 2008), lo cierto es que el uso de la red con fines sexuales se ha relacionado también con la aparición de nuevas formas de adicción. La adicción al cibersexo describiría el uso excesivo e incontrolado de Internet con objetivos de gratificación sexual, un perfil asociado a múltiples efectos negativos (como problemas de pareja, personales, laborales, etc) (Voros, 2009; Daneback, Cooper \& Mansson, 2005), más concretamente, se ha propuesto que la adicción a la pornografía online sería una de las OSAs con mayor potencial adictivo (Griffiths, 2010).

La búsqueda de pornografía y su visualización se tratan de la principal actividad sexual entre los usuarios de internet (Griffiths, 2010; Dorton \& Gast, 2007), así, en torno al 76\% de los usuarios de Internet consumen 0 han consumido pornografía (Griffiths, 2012). De acuerdo con Laier, Pawlikowski, Pekal, Schulte \& Brand (2013), la pornografía se trata de una actividad sexual online solitaria y cuya finalidad se circunscribe al entretenimiento y gratificación, lo que se relacionaría con un elevado riesgo adictivo y lo que propiciaría un consumo problemático y excesivo (Young, 2008). Entre los estudios que avalan este perfil, se encuentra el estudio de Meerkerk, Van Den Eijnden \& Garretsen (2006), en el que se describe una correlación positiva entre el consumo previo de pornografía y tendencias adictivas posteriores o el de Serrano \& Cuesta (2018), que apuntan que el perfil problemático de internet se vincula a la pornografía y su búsqueda.

La capacidad adictiva de la pornografía se debe en parte a una serie de factores comunes a cualquier forma de adicción a Internet: anonimato, accesibilidad y comodidad/coste (Modelo de la Triple A) (Young, 2008). El anonimato que ofrece internet lleva al usuario a moverse en un entorno seguro en el que puede experimentar y explorar su sexualidad sin límites y sin temor a ser juzgado, y en ello, la pornografía puede tomar un papel central en la búsqueda de identidades y experiencias (Chaney \& Dew, 2003). Por otro lado, en internet se encuentra accesible gran variedad de contenidos de forma sencilla y libre de coste alguno (Laier, Pawlikowski, Pekal, Schulte \& Brand, 2013; Chaney \& Dew, 2003). Además y de acuerdo con Young (1999), el sexo online en general, y la por- 
nografía en concreto pueden ser una herramienta que el sujeto use para evadirse de la realidad y sus problemas, lo que le resulta altamente gratificante y reforzador (Ballester, 2006)

La adicción al cibersexo se caracteriza por un esfuerzo reiterado e infructuoso por reducir, controlar o eliminar el consumo, por una pérdida de control sobre la conducta y por una dedicación excesiva (más de 11 horas a la semana) que genera un menoscabo en otras áreas importantes de la vida 0 limita la atención a otras responsabilidades (Wéry \& Billieux, 2017). En otras adicciones tóxicas 0 conductuales, en la adicción al cibersexo en general y a la pornografía en particular se manifiestan síntomas como la saliencia de la actividad adictiva (obsesión hacia el sexo en Internet), la realización de la actividad a fin de regular las emociones, tolerancia, síndrome de abstinencia, conflictos en diferentes áreas y recaída (Griffiths, 2010). Todo ello, ha favorecido el interés por estudiar aquellos factores que pueden actuar como predisponentes o mantenedores del problema.

Sin lugar a dudas, uno de los síntomas que más atención acapara es el mantenimiento del consumo a pesar de las consecuencias negativas que éste genera (Grubbs, Volk, Exline \& Pargament, 2015; Griffiths, 2012; Ballester, 2006). De modo que algunos estudios han tratado de explorar los motivos que explican esta conducta, señalando entre otros aspectos, la capacidad del contenido pornográfico para desviar la atención de otros estímulos tan importantes como son los problemas que se derivan del consumo (Laier, Pawlikowski, Pekal, Schulte \& Brand 2013; Laier, Schulte \& Brand, 2013; Macapagal, Janssen, Friedberg, Finn \& Heiman, 2011).

El sexo resulta para el ser humano un estímulo extremadamente saliente per se, de manera que su procesamiento se prioriza respecto al procesamiento de otras claves contextuales y estimulares (Griffiths, 2012; Griffiths, 2010). Dicha saliencia se ve potenciada por la capacidad de los estímulos sexuales para generar arousal, es decir, un grado de activación sexual elevado (Laier, Schulte \& Brand, 2013). La activación sexual, que se ha erigido como predictor del uso problemático de internet con fines sexual y del consumo de pornografía (Wéry \& Billieux, 2017; Laier, Pawlikowski, Pekal, Schulte \& Brand, 2013), junto a la saliencia del sexo pueden tener efecto sobre factores cognitivos y ejecutivos, interfiriendo en otras tareas y en el procesamiento de otros estímulos. Esto es precisamente lo que se documenta en el estudio de Macapagal et.al. (2013), en el que a través de una tarea Go/No Go paralela a la visualización de contenido pornográfico se registraron efectos significativos en funciones cognitivas como la atención y ejecución. Concretamente, en este estudio lo que se halla es que existe efectos claros y significativos sobre la ejecución en la tarea Go/No Go en aqueIlos participantes que presentaban elevados niveles de excitación sexual, los que presentaban mayor tasa de error y omisión. Es decir, aquellas personas con mayor arousal sexual presentaban peor rendimiento cognitivo

De esta manera, el contenido pornográfico en sí mismo parecería tener la capacidad para atraer y consumir recursos atencionales, generando una interferencia en la atención y el procesamiento de otros estímulos relevantes. Por todo ello, el objetivo del presente estudio sería explorar la relación entre la interferencia atencional generada por el contenido pornográfico y el consumo y la gravedad de sus consecuencias. A tal fin, se realizó una tarea experimental en la que se presentaron videos tanto de contenido pornográfico como de otros tipos (series de televisión, videojuegos 0 documentales), de manera paralela a la presentación y demanda de ejecución de una tarea atencional Stroop (similar en términos de procesos atencionales subyacentes a la tarea Go/No Go anteriormente citada). La hipótesis de partida para este trabajo es que los participantes mostrarán tiempos de reacción mayores y menor cantidad de aciertos durante la tarea Stroop paralela a la visualización del video con contenido pornográfico, sobre todo cuando se compare con el video de bajo contenido interactivo. 


\section{MÉTODO}

\section{Participantes}

En este estudio participaron un total de 58 personas, de las cuales 32 eran mujeres (55.17\%) y 26 hombres (44.83\%). Todos ellos tenían edades comprendidas entre los 18 y 35 años con una edad media de 24.29 años $(S D=3.40)$. A la hora de seleccionar la muestra, los criterios de inclusión utilizados fueron: tener una edad superior a 18 años e inferior a 35, y no mostrar síntomas de trastorno mental que puedan producir una alteración en los procesos atencionales (p.e. trastorno depresivo mayor) y consentir ser expuesto a contenidos sexuales.

\section{Procedimiento e instrumento}

Los participantes fueron reclutados mediante diferentes vías: contacto directo e indirecto (método bola de nieve), mensajes y anuncios en diferentes redes sociales y apps de comunicación (Whatsapp, Facebook e Instagram), etc. Todos ellos fueron citados de forma individual y presencial entre noviembre del 2018 y marzo del 2019. Los participantes eran introducidos en un laboratorio de investigación carente de estimulación que pudiera resultar distractora y sin referencias temporales. La finalidad de eliminar toda referencia temporal era evitar la falsificación de datos durante el estudio, ya que una de las tareas experimentales tenía que ver con la precisión en la estimación de intervalos temporales (tarea no analizada en este trabajo). Una vez en la sala, se les daba una explicación básica sobre el procedimiento a seguir y se resolvían sus dudas. Tras ello, los participantes se quedaban solos en la sala durante la duración íntegra de la condición experimental, con objeto de preservar su privacidad.

La tarea experimental de Interferencia se basaba en el paradigma Stroop clásico de medida de tiempos de reacción. En concreto, esta tarea experimental se dividía en dos secciones: un ensayo inicial de práctica que incluía además una medida de control y cuatro ensayos experimentales. El ensayo de práctica se realizaba con el objetivo de que el sujeto se acostumbrara antes de realizar el ensayo control y los ensayos experimentales; así, durante este ensayo, los participantes completaban la tarea Stroop sin ningún tipo de distractor y a una velocidad inferior que facilitara el aprendizaje del paradigma. Una vez familiarizado, el paciente completaba la tarea Stroop nuevamente sin ningún distractor pero a velocidad normal (condición control). Posteriormente, se iniciaban los ensayos experimentales. Durante estos ensayos, los sujetos eran expuestos a un video de cada uno de los contenidos multimedia comentados anteriormente, es decir, pornografía (condición porno), series de televisión (condición serie deTV), videojuegos (condición videojuego) y un documental de un hombre leyendo el periódico (condición documental), mientras que de manera paralela en la parte superior derecha de la pantalla aparecía un cuadro donde se exponía la Tarea Stroop a realizar. Esta tarea Stroop estaba compuesta únicamente por ensayos incongruentes, es decir, el color de la palabra no coincide con lo que pone. Los sujetos recibían la premisa de ignorar el video y centrarse en la realización de la tarea lo más rápida y precisa posible. Al responder, el sistema registraba varios parámetros: tiempos de reacción (TR), aciertos, errores y las omisiones en caso de no responder. La finalidad de la tarea era evaluar las diferencias en el tiempo de reacción en función del contenido multimedia al que los sujetos eran expuestos, es decir, los recursos atencionales que consumía cada tipo de contenido.

\section{RESULTADOS}

Con el objetivo de explorar y evaluar el grado de significación de las diferencias en los tiempos de reacción (en milisegundos -ms-) y en el porcentaje de respuestas correctas se estimó un modelo lineal general (GLM) de medidas repetidas. A través de este modelo, se encontraron que se regis- 
tran tiempos medios de reacción claramente superiores durante la realización de la tarea Stroop al mismo tiempo que se exponía pornografía ( $M=0.9033, S D=0.1641)$, seguida por las condiciones de exposición paralela a: series de televisión $(M=0.8818, S D=0.2827)$, documental $(M=0.7959$, $S D=0.1995)$ y videojuego $(M=0.7717, S D=0.1527)$. Las diferencias resultan especialmente significativas cuando se comparan con la condición control, dónde se registraron los tiempos medios de reacción más bajos ( $M=0.7445, S D=0.1641)$. Analizada en conjunto, la diferencia en los TR en función de la condición experimental alcanzaba la significación estadística (Wilks' =.486; F=14.01; $p<$ .001). Los tiempos de reacción de cada condición experimental se registran en la figura 1.

Figura 1. Tiempo de reacción durante la tarea Stroop en las distintas condiciones

\section{Tiempo medio de reacción (en milisegundos)}

1

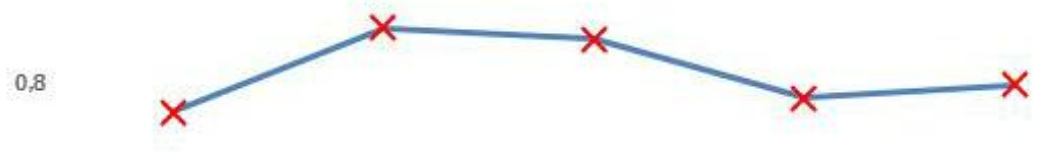

0,6

0,4

0,2

0

Condicióncontrol $\begin{gathered}\text { Condición } \\ \text { pornografia }\end{gathered} \quad$ Condiciónserie TV $\quad \begin{gathered}\text { Condición } \\ \text { videojuego }\end{gathered} \begin{gathered}\text { Condición } \\ \text { documental }\end{gathered}$

Asimismo, se llevó a cabo una comparación Bonferroni post-hoc a fin de comparar las condiciones por pares y ver entre qué condiciones las diferencias eran significativas. Así, se encontró que los tiempos de reacción eran significativamente superiores durante la condición de pornografía respecto a la control $(p<.001)$, la de videojuegos $(p<.001)$ y la de bajo contenido multimedia $(p<.001)$, pero no la de series de televisión ( $p=.394)$.

En cuanto al porcentaje de aciertos a la hora de responder a los estímulos Stroop (figura 2), encontramos que el mayor número de aciertos (y por tanto, menor cantidad de fallos), se daba en la condición de visionado de videojuegos (92.02\%), seguido de la condición control (sólo Stroop) $(91.16 \%)$, la de pornografía (88.50\%), documental de bajo contenido multimedia (88.29\%), y finalmente, las series de televisión (86.20\%). Sin embargo, y de acuerdo a lo encontrado en el modelo lineal general de medidas repetidas, las diferencias en el número de aciertos no eran estadísticamente significativas. 
Figura 2. Respuestas correctas (\%) durante la tarea Streop en las distintas condiciones

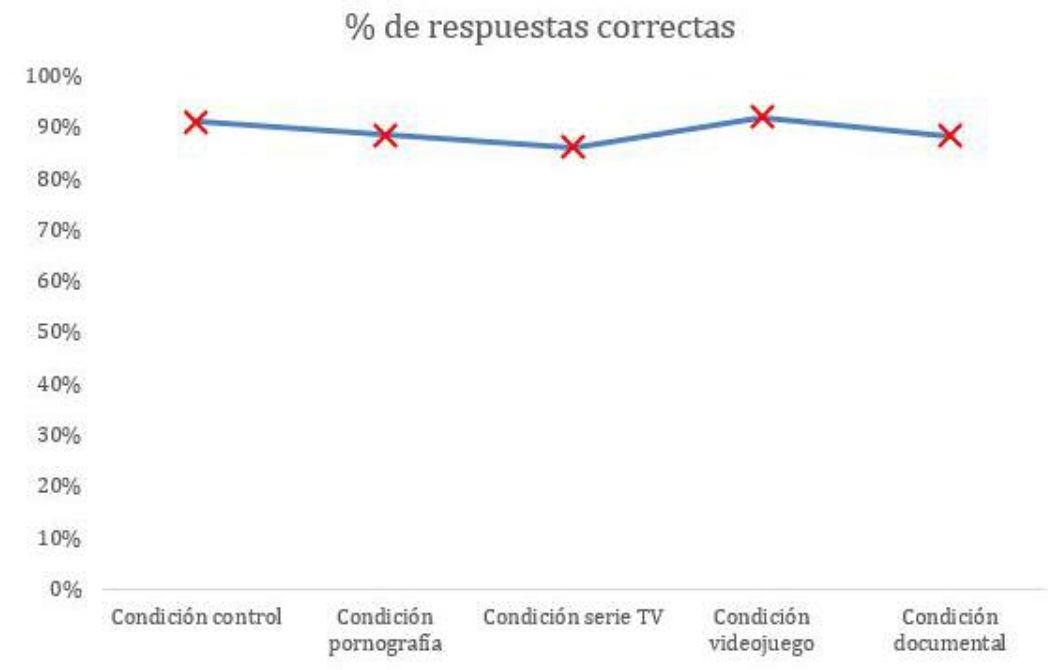

A modo de resumen, en la tabla siguiente (tabla 1), se muestra los datos concretos respecto a los tiempos medios de reacción en ms, y el número y porcentaje de aciertos y fallos en las distintas condiciones experimentales.

Tabla 1. Tiempo de reacción, \% de respuestas correctas y no correctas (incorrectas/omisiones) en distintas condiciones experimentales

\begin{tabular}{|c|c|c|c|}
\hline & $\begin{array}{c}\text { Tiempos de } \\
\text { reacción } \\
X \text { en milisegundos } \\
(S D)\end{array}$ & $\begin{array}{l}\text { Respuestas } \\
\text { correctas } \\
\mathrm{n}(\%)\end{array}$ & $\begin{array}{c}\text { Respuestas no } \\
\text { correctas } \\
\mathrm{n}(\%)\end{array}$ \\
\hline Condición control (sólo tarea Stroep) & $0.7445(.1641)$ & $21.88(91.16 \%)$ & $2.12(8.84 \%)$ \\
\hline $\begin{array}{l}\text { Condición pornografia (tarea Strosp + video } \\
\text { pornográfico) }\end{array}$ & $0.9033(.2379)$ & $21.24(88.50 \%)$ & $2.76(11.49 \%)$ \\
\hline $\begin{array}{l}\text { Condición serie de TV (tarea Stroop + video } \\
\text { serie de TV) }\end{array}$ & $0.8818(.2827)$ & $20.69(86.20 \%)$ & $3.31(13.59 \%)$ \\
\hline $\begin{array}{l}\text { Condición videojuego (tarea Stroop + vídeo } \\
\text { videojuego) }\end{array}$ & $0.7717(.1527)$ & $22.09(92.02 \%)$ & $1.91(7.97 \%)$ \\
\hline $\begin{array}{l}\text { Condición documental (tarea Stroop + vídeo } \\
\text { documental) }\end{array}$ & $0.7959(.1995)$ & $21.19(88.29 \%)$ & $2.81(11.70 \%)$ \\
\hline
\end{tabular}

\section{DISCUSIÓN}

A lo largo de este estudio se ha pretendido explorar la capacidad de las claves sexuales, en este caso en forma de videos pornográficos, para interferir y afectar en la atención y procesamiento de otras claves estimulares: es decir, el efecto que la pornografía puede llegar a generar en diversas y variadas funciones cognitivas y ejecutivas, con el objeto de explicar la perseverancia y la incapacidad de interrumpir el consumo y la conducta que están generando una serie de conflictos y problemas en la vida del adicto al sexo online. En concreto, se ha tratado de trazar y dibujar la relación existente entre la interferencia atencional que puede generar la pornografía y la gravedad del con- 
sumo de éste, para lo que se ha utilizado un paradigma ampliamente validado y replicado como es el de la tarea atencional Stroop. Para ello, 58 participantes con edades comprendidas entre los $18 \mathrm{y}$ 35 años fueron reclutados y realizaron una tarea experimental ad hoc para evaluar este efecto. La selección de este rango de edad no fue aleatoria, sino que varios estudios apuntan a que la población joven se encuentra más vinculada a todo tipo de OSAs (Egan \& Parmar, 2013; Meerkerk, Van Den Eijnden \& Garretsen, 2006), de modo que evaluar este sector de población resultaría más ecológico de cara a extrapolar y generalizar los resultados.

Los datos obtenidos en este estudio apoyan la hipótesis de partida de manera parcial. Por un lado, se ha confirmado que los tiempos de reacción son significativamente mayores durante la tarea Stroop concurrente con la visualización del contenido pornográfico, lo que significa que la pornografía efectivamente genera una interferencia cognitiva clara. Estos resultados van en la línea de los resultados obtenidos por Macapagal et. al. (2011) en su estudio, en el que utilizando el paradigma Go/NoGo ya se detectó una clara influencia de los estímulos sexuales en el peor procesamiento y menores niveles de atención dedicados a otros estímulos también importantes pero presumiblemente no tan salientes como el sexo. Estos datos se podrían explicar aludiendo a la gran activación 0 arousal que generan las claves sexuales asociadas a su saliencia y potencia subjetivas (Wéry \& Billieux, 2017; Laier, Pawlikowski, Pekal, Schulte \& Brand, 2013). Sin embargo, no se ha encontrado que el contenido pornográfico influya en el número de aciertos, fallos u omisiones, lo que se había hipotetizado en un inicio y no se ha podido confirmar, ya que las diferencias encontradas no son estadísticamente significativas.

Aunque no se ha analizado de forma exhaustiva, a la luz de los datos obtenidos parece ser que la capacidad para captar recursos atencionales no solo se da en la pornografía, sino que también se da, en menor medida y de forma moderada, en otros contenidos que resultan ricos e interactivos para los sujetos, como son las series de televisión, posiblemente por ser fuente de estimulación, entretenimiento y de evocación de emociones y estados mentales. Siguiendo esta premisa cabría esperar lo mismo de los videojuegos, pero en este estudio no se ha encontrado una gran capacidad de recaptación atencional por parte de éstos. Una posible explicación es que los videojuegos en sí mismos no tienen hilo argumental que permitan al espectador introducirse en ellos con su simple visualización, por lo que no resultarían muy interactivos para los sujetos. Es decir, que si en lugar de un video sobre videojuegos, los participantes hubieran tenido la oportunidad de jugar (es decir, exponerse a la capacidad plenamente inmersiva de los videojuegos), muy probablemente los resultados hubieran sido significativamente diferentes.

Una fortaleza de este estudio es que en los participantes se ha hecho inclusión de ambos sexos, lo que resultaría relativamente novedoso, puesto que la investigación tradicional en compulsión y adicción sexual se ha orientado a un colectivo masculino (presumiblemente) por presentar mayores prevalencias de éstas, dejando al colectivo femenino de lado. Por ello, una posible línea de estudio futura podría ser la replicación de este mismo estudio atendiendo también a las diferencias por sexos en el impacto del contenido pornográfico en las funciones cognitivas. Para ello, y con objeto de obtener mayor robustez y peso en los hallazgos, convendría ampliar la muestra actual en ambos sexos.

\section{REFERENCIAS BIBLIOGRÁFICAS}

Ballester, R. (2006). Tratamiento psicológico de un caso de adicción al cibersexo. En F.X. Méndez, J.P. Espadà y Orgiles: Terapia Psicológica en niños y adolescentes: Estudio de Casos prácticos, 387-414, Madrid: Pirámide

Chaney, M. P., \& Dew, B. J. (2003). Online experiences of sexually compulsive men who have sex 
with men. Sexual Addiction and Compulsivity, 10(4), 259-274.

Cooper, A., Scherer, C.R., Boies, S.C. \& Gordon, B.L. (1998). Sexuality on the Internet.

Cooper, A., McLoughlin, I.P. \& Campbell, K.M. (2000). Sexuality in Cyberspace: Update for the 21st century. CyberPsychology \& Behavior, 3(4), 521-536

Daneback, K., Cooper, A. \& Mansson, S.A. (2005). An Internet Study of Cybersex Participants. Archives of Sexual Behavior, 34(3), 321-328

Egan, V., \& Parmar, R. (2013). Dirty habits? Online pornography use, personality, obsessionality, and compulsivity. Journal of Sex \& Marital Therapy, 39(5), 394-409.

Griffiths, M.D. (2000). Excessive Internet Use: Implications for Sexual Behavior. CyberPsychology \& Behavior, 3(4), 537-551

Griffiths, M.D. (2010). Sex on the internet: observations and implications for internet sex addiction. Journal of sex research, 38(4), 333-342

Griffiths, M.D. (2012). Internet sex addiction: A review of empirical research. Addiction Research and Theory, 20, 111-124.

Grov, C., Bamonte, A., Fuentes, A., Parsons, J. T., Bimbi, D. S., \& Morgenstern, J. (2008). Exploring the Internet's role in sexual compulsivity and out of control sexual thoughts/behavior: A qualitative study of gay and bisexual men in New York City. Culture, Health \& Sexuality, 10(2), 107-124

Grubbs, J.B., Volk, F., Exline, J.J. \& Pargament, K.I. (2015). Internet Pornography Use: Perceived Addiction, Psychological Distress, and the Validation of a Brief Measure. Journal of Sex \& Marital Therapy, 41(1), 86-103

Laier, C., Schulte, F. P., \& Brand, M. (2013). Pornographic picture processing interferes with working memory performance. Journal of Sex Research, 50(7), 642-652

Laier, C., Pawlikowski, M., Pekal, J., Schulte, F.P. \& Brand. M. (2013). Cybersex addiction: Experienced sexual arousal when watchin gpornography and not real-life sexual contacts makes the difference. Journal of Behavioral Addictions, 2(2), 100-107.

Macapagal, K. R., Janssen, E., Fridberg, D. J., Finn, P. R., \& Heiman, J. R. (2011). Theeffects of impulsivity, sexual arousability, and abstract intellectual ability on men's and women's go/no-go task performance. Archives of Sexual Behavior, 40(5), 995

McCullough, B. (Producer). (2015, January 04). A history of Internet porn [Transcrito de un audio podcast].Extraído de: http://www.internethistorypodcast.com/

Meerkerk, G., Van Den Eijnden, R.J.J.M. \& Garretsen, H.F.L. (2006). Predicting Compulsive Internet Use: It's All About Sex!. Cyberpsychology \& Behavior, 9(1), 95-103.

Serrano, 0. \& Cuesta, V. (2018). Propuesta de variables para el diseño de un modelo sobre TECNOADICCIÓN al sexo en la población juvenil. Revista Española de Comunicación en Salud, 9(1), 6471

Young, K.S. (2008). Internet Sex Addiction: Risk Factors, Stages of Development and Treatment. American Behavioral Scientist 52(1), 21-37 
